\title{
Accuracy Analysis and Structure Configuration of Binocular Vision System
}

\author{
Xinfeng Fan ${ }^{a}$, Yuanzeng Cheng and Qiang Fu \\ Ordance Engineering College, Shijiazhuang 050003, China \\ anichowen@sina.com
}

Keywords: vision measurement, binocular vision, structure configuration, accuracy analysis.

\begin{abstract}
In order to improve the measurement accuracy of binocular vision system, a structural model is build. On the basis of it, relational features between target point and structural parameter of system are analyzed, and the effect of each parameter to the measurement accuracy is simulated. According to simulation results, the general principles and methods of structure configuration for binocular vision system is offered.
\end{abstract}

\section{Introduction}

Vision measurement is an advanced system based on computer vision. Due to non-contact vision measurement, high efficiency, quick reaction, etc., now it has been widely used in modern processing industry. Traditional measuring equipment such as micrometer, meter stick cannot meet the requirements of modern processing industry, thus more and more experts and scholar study efficiently and accurately vision measurement technology.

Binocular vision system is the most common vision sensor configuration in vision measurement. Its basic principle is that it calculates image parallax of a target to obtain three-dimensional information of it by collecting images of two cameras at different locations. The simple binocular vision system has the advantage of real-time online measurement, so it has wide application prospect in the future of industrial measurement. But the measurement accuracy is influenced such as the precision of camera calibration and image matching, external lighting, architecture. At the same time, the current measurement precision is unable to meet the industrial measurement needs, so how to improve the measurement accuracy of binocular vision system has become an important issue to be studied. Generally, the system uses human experience [1, 2] to be built. At the same time, the current research of binocular vision system is focused on camera calibration [3], image matching [4] and other important aspects, lacking of systematic measurement accuracy analysis. Thus, the study of the structure of binocular vision system configuration and precision analysis is important, helping to improve the measurement accuracy and its application.

This paper analyzes the influence of structural parameters to measurement accuracy, according to the structure model of binocular vision system, which reflects the relationship between space point and imaging point. And the structure configuration is get according to the simulation results.

\section{Structure Model of Binocular Vision System}

This paper builds a most common structure model of binocular vision system as shown in Fig. 1 . To facilitate the analysis, the original coordinate point is established in the projecting center of left CCD camera. The effective focal length of the left and right two cameras, whose projection center are $o_{c 1}$ and $o_{c 2}$, are $f_{1}$ and $f_{2}$. The distance between projecting centers are baseline distance $B . o_{1} x_{1} y_{1}$ and $o_{2} x_{2} y_{2}$ is the image plane coordinate system, and $o_{c 1} x_{c} y_{c} z_{c}$ is the camera coordinate system. In the space, there is a point $P\left(x_{c}, y_{c}, z_{c}\right)$, whose projecting point is $p_{1}\left(x_{1}, y_{1}\right)$ and $p_{2}\left(x_{2}, y_{2}\right)$. $\omega_{1}$ and $\omega_{2}$ is horizontal viewing angle between projecting line and the optical axis. $\beta_{1}$ and $\beta_{2}$ is vertical viewing angle between projecting line and the target line. 


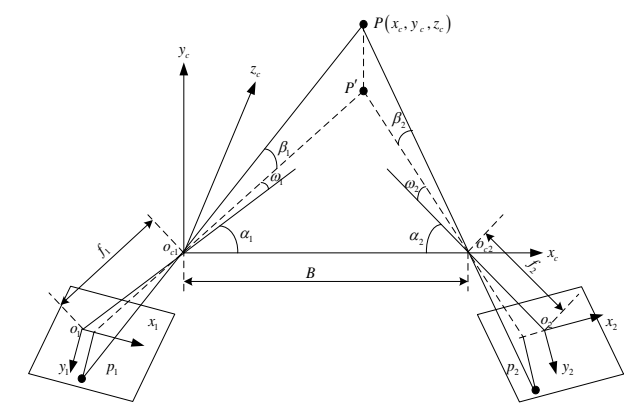

Fig. 1 Structure model of binocular vision system

According to geometry relationship in Fig.1, there is an expression as show in (1). Then the three-dimensional coordinates of point $P$ is expressed as (2). In (2), $\omega_{1}$ and $\omega_{2}$ meets the pinhole imaging principle, $\omega_{1}=\arctan \left(x_{1} / f_{1}\right), \omega_{2}=\arctan \left(x_{2} / f_{2}\right)$.

$$
\begin{aligned}
& \left\{\begin{array}{l}
x_{c}=z_{c} \cot \left(\alpha_{1}+\omega_{1}\right) \\
B=z_{c} \cot \left(\alpha_{1}+\omega_{1}\right)+z_{c} \cot \left(\alpha_{2}+\omega_{2}\right) \\
\tan \beta_{1}=\frac{y_{c}}{z_{c} / \sin \left(\alpha_{1}+\omega_{1}\right)} \\
\tan \beta_{2}=\frac{y_{c}}{z_{c} / \sin \left(\alpha_{2}+\omega_{2}\right)} \\
\tan \beta_{1}=\frac{y_{1} \cos \omega_{1}}{f_{1}}, \tan \beta_{2}=\frac{y_{2} \cos \omega_{2}}{f_{2}}
\end{array}\right. \\
& \left\{\begin{array}{l}
x_{c}=\frac{B \cot \left(\alpha_{1}+\omega_{1}\right)}{\cot \left(\alpha_{1}+\omega_{1}\right)+\cot \left(\alpha_{2}+\omega_{2}\right)} \\
y_{c}=\frac{B \sin \left(\alpha_{2}+\omega_{2}\right) \tan \beta_{1}}{\sin \left[\left(\alpha_{1}+\omega_{1}\right)+\left(\alpha_{2}+\omega_{2}\right)\right]} \\
=\frac{B \sin \left(\alpha_{1}+\omega_{1}\right) \tan \beta_{2}}{\sin \left[\left(\alpha_{1}+\omega_{1}\right)+\left(\alpha_{2}+\omega_{2}\right)\right]} \\
z_{c}=\frac{B}{\cot \left(\alpha_{1}+\omega_{1}\right)+\cot \left(\alpha_{2}+\omega_{2}\right)}
\end{array}\right.
\end{aligned}
$$

\section{Accuracy Analysis of Binocular Vision System}

According to the structure model, the three-dimensional coordinate of space point not only depends on its image coordinates, but also depends on the effective focal length, baseline distance, the angle between the optical axis and the baseline, the viewing angle, etc. Vector expression of (2) is shown as in (3).

$$
\begin{aligned}
& P\left(x_{c}, y_{c}, z_{c}\right)=F\left(x_{1}, y_{1}, x_{2}, y_{2}, f_{1}, f_{2}, B, \alpha_{1}, \alpha_{2}, \omega_{1}, \omega_{2}, \beta_{1}, \beta_{2}\right) \\
& \Delta=\sqrt{\left(\Delta x_{c}\right)^{2}+\left(\Delta y_{c}\right)^{2}+\left(\Delta z_{c}\right)^{2}}=\sqrt{\sum_{j} \sum_{i}\left(\frac{\partial F}{\partial i} \delta i\right)^{2}}
\end{aligned}
$$

The overall measurement error function [5] is shown as in (4). $i=x_{1}, y_{1}, x_{2}, y_{2}, f_{1}, f_{2}, B, \alpha_{1}, \alpha_{2}$, $\omega_{1}, \omega_{2}, \beta_{1}, \beta_{2}$ is the impact factor. $j=x_{c}, y_{c}, z_{c}$ is the three-dimensional direction of point $P . \frac{\partial F}{\partial i}$ is the error transfer function of impact factor. $\delta i$ is the error of impact factor. In the following section, the main factor will be analyzed including the angle between the optical axis and the baseline, the effective focal length and baseline distance.

\subsection{The influence of the angle $\alpha$ between the optical axis and the baseline.}

The angle $\alpha$ is an important parameter affecting the placement of the two cameras. To facilitate analysis, assuming a symmetrical structure, some parameters simplify as follows, $\alpha_{1}=\alpha_{2}=\alpha$, 
$f_{1}=f_{2}=f, \omega_{1}=\omega_{2}=\omega, \delta x_{1}=\delta x_{2}=\delta y_{1}=\delta y_{2}=\delta, \beta_{1}=\beta_{2}=\beta, \alpha+\omega=\lambda$. Then combing (2) $\sim(4)$, the overall measurement error function of image coordinates is calculated as (5).

$$
\Delta_{x y}=\frac{\sqrt{2} B \delta}{f} \frac{\cos \omega}{\sin 2 \lambda} \sqrt{\sin ^{2} \lambda+\frac{\cos ^{2} \omega}{4 \cos ^{2} \lambda}+\tan ^{2} \beta\left(\frac{\cos \omega}{2 \cos \lambda}+\cos \alpha\right)^{2}}
$$

With the increase of vertical viewing angle, the overall measurement error $\Delta_{x y}$ is monotonically increasing. So, the vertical viewing angle is assumed as $\beta=30^{\circ}$. When $\omega=-30^{\circ} \sim 30^{\circ}$ and $\alpha=15^{\circ}+5^{\circ} * k(k=1,2 \cdots 11,12)$, the measurement error curve distribution between $\Delta_{x y} f /(\sqrt{2} B \delta)$ and $\alpha$ is shown in Fig. 2.

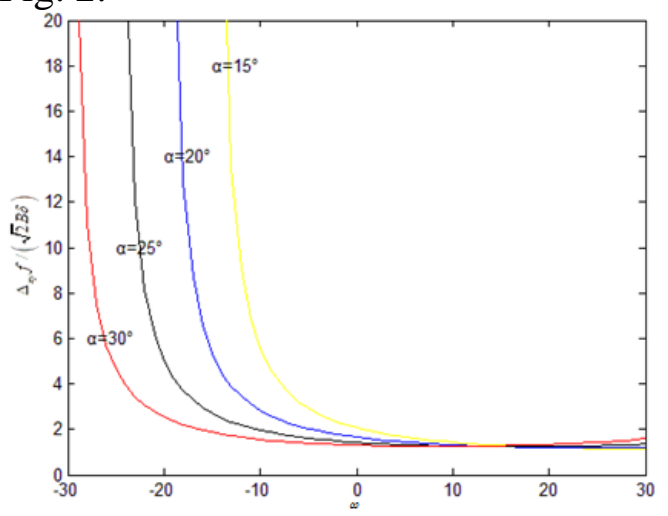

(a) $\alpha$ is varying in $15^{\circ} \sim 30^{\circ}$

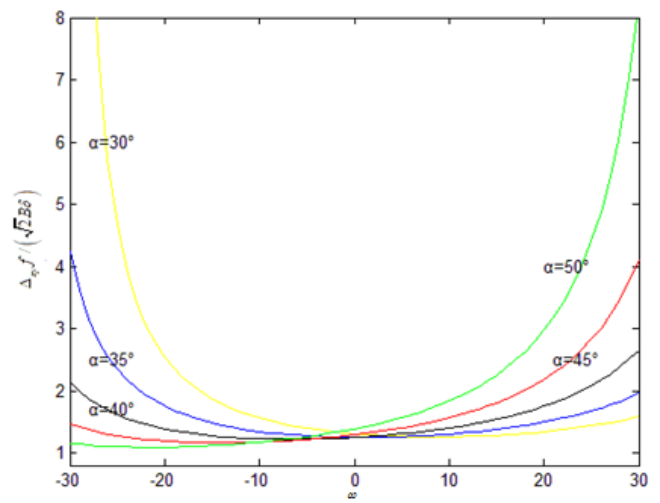

(b) $\alpha$ is varying in $30^{\circ} \sim 50^{\circ}$

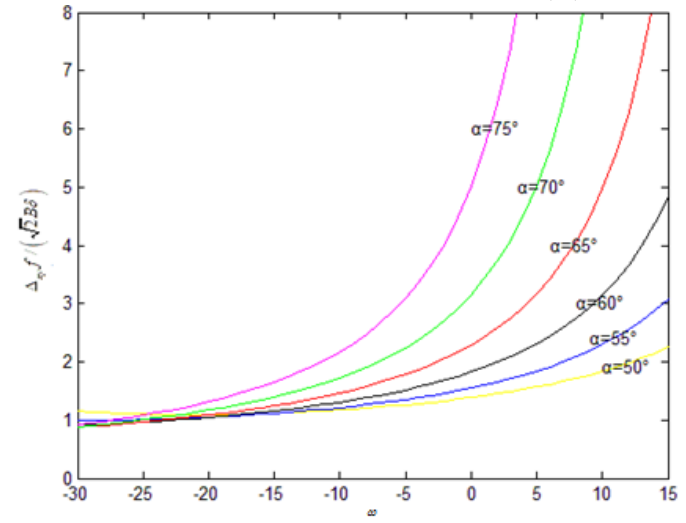

(c) $\alpha$ is varying in $50^{\circ} \sim 75^{\circ}$

Fig. 2 The measurement error curve distribution of $\alpha$

According to the measurement error curve distribution in Fig. 2, the error is different when the angle $\alpha$ has a different value. In (b), when $\alpha$ is varying in $30^{\circ} \sim 50^{\circ}$, measurement error is changing smoothly over the entire field of view, and it has a minimum value. In (a), when $\alpha$ is varying in $15^{\circ} \sim 30^{\circ}$, measurement error is changing obviously over the entire field of view, and it increases sharply with the decrease of $\alpha$ at the direction of the negative horizontal field angle $\omega$. In (c), when $\alpha$ is varying in $50^{\circ} \sim 75^{\circ}$, measurement error is changing obviously over the entire field of view, and it increases sharply with the increase of $\alpha$ at the direction of the positive horizontal field angle $\omega$. So, a better binocular vision system should keep the angle $\alpha$ varying in $30^{\circ} \sim 50^{\circ}$, and the error measurement accuracy is the best.

\subsection{The influence of the effective focal length $f$.}

To facilitate analysis, assuming a symmetrical structure, some parameters simplify as follows, $f_{1}=f_{2}=f, \alpha_{1}=\alpha_{2}=\alpha, \omega_{1}=\omega_{2}=\omega, \delta f_{1}=\delta f_{2}=\delta f=\delta, \beta_{1}=\beta_{2}=\beta, \alpha+\omega=\lambda$. According to expression (2), the error transfer function of the effective focal length is calculated as (6). Combining (3) and (4), the overall measurement error function of effective focal length is calculated as (7). 


$$
\begin{aligned}
& \left.\frac{\partial F}{\partial f}=\frac{B \sin \lambda \sin \omega}{f \sin ^{2}(2 \lambda)} \sqrt{\cos ^{2} \omega+\left\{\begin{array}{l}
\sin \lambda \tan \beta[\sin (2 \lambda) \cos \alpha-\sin \lambda \cos \omega] \\
\sin ^{2} \lambda \sin (2 \lambda) \tan \beta
\end{array}\right.}\right\}^{2} \\
& \Delta_{f}=\sqrt{2\left(\frac{\partial F}{\partial f} \delta f\right)^{2}}=\sqrt{2} \delta \frac{\partial F}{\partial f}
\end{aligned}
$$

Assuming $\omega=10^{\circ}, \beta=30^{\circ}$, when the angle between the optical axis and the baseline vary in $30^{\circ} \sim 50^{\circ}$, the measurement error curve distribution between $\Delta_{f} /(\sqrt{2} B \delta)$ and $f$ is shown in Fig. 3.

According to the measurement error curve distribution in Fig. 3, the error decreases with the increase of $f$. Because measurement error decreases with the (horizontal) viewing angle, so the conclusion that if the effective focal length increases, the viewing angle will decrease, at the same time, the measurement accuracy increases, can be get. According to principles of optics [6], the viewing angle will decrease if the effective focal length increases. So, in the measuring range, the error can be reduced to improve the accuracy of measurement by increasing the effective focal length of the system.

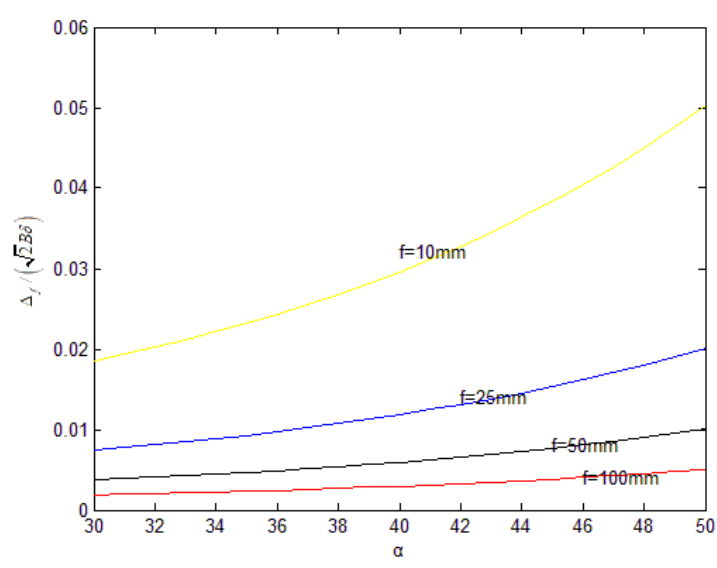

(a) Distribution of $f$

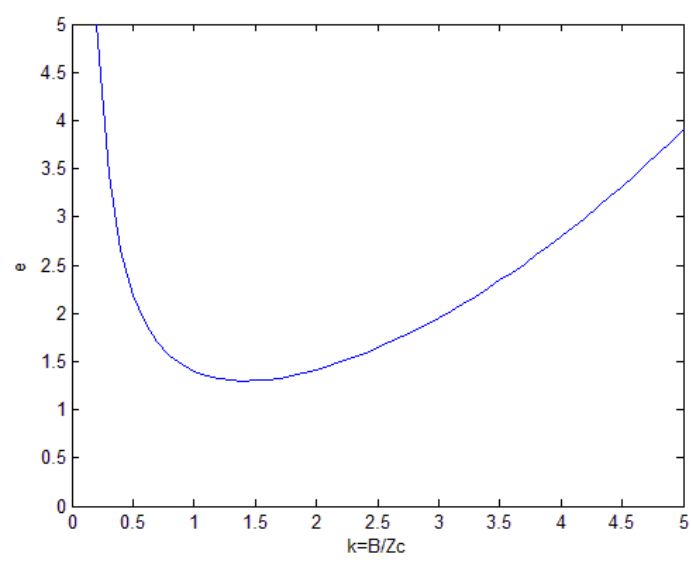

(b) Distribution of $k=B / z_{c}$

Fig. 3 The measurement error curve distribution of $f$ and $k=B / z_{c}$

\subsection{The influence of the baseline distance $B$.}

The baseline distance characterizes the positional relationship between the two cameras directly, determining the measurement accuracy of the system. The influence of baseline distance is complex. When it increases, the corresponding measurement angle $\alpha+\omega$ becomes large, so that the influence of it is nonlinear. According to the analysis above, when the system is a symmetrical structure and the viewing angle is zero, the accuracy of the system is the highest. Assuming $\alpha_{1}=\alpha_{2}=\alpha$, $\omega_{1}=\omega_{2}=\beta_{1}=\beta_{2}=0, f_{1}=f_{2}=f$, so $y_{c}=0$ (the system has no error at axis $y_{c}$ ).

According to (2), the error transfer function of the image coordinates is calculated as (8). Assuming $\delta x_{1}=\delta x_{2}=\delta y_{1}=\delta y_{2}=\delta$, combining (3) and (4), the overall measurement error function of the image coordinates is calculated as (9).

$$
\begin{aligned}
& \left\{\begin{array}{l}
\frac{\partial x_{c}}{\partial x_{1}}=\frac{\partial x_{c}}{\partial x_{2}}=-\frac{z_{c}^{2}}{B f} \frac{\cot \alpha}{\sin ^{2} \alpha} \\
\frac{\partial z_{c}}{\partial x_{1}}=\frac{\partial z_{c}}{\partial x_{2}}=\frac{z_{c}^{2}}{B f \sin ^{2} \alpha} \\
\Delta_{B}=\sqrt{2\left(\frac{\partial x_{c}}{\partial x_{1}} \delta\right)^{2}+2\left(\frac{\partial Z_{c}}{\partial x_{1}} \delta\right)^{2}}=\frac{\sqrt{2} z_{c}^{2} \delta}{B f \sin ^{3} \alpha}
\end{array}\right.
\end{aligned}
$$


According to geometry relationship and assumption, the expression $\sin \alpha=\frac{Z_{c}}{B / 2}$ is found. If $k=B / z_{c}$, then the relationship between $\Delta_{B}$ and $k$ is calculated as in (10). There are there constant $z_{c}$, $\delta, f$ in (10) for a known system and target, so $\Delta_{B} \propto e$, and error $e$ can describes error $\Delta_{B}$. The measurement error curve distribution between $e$ and $k=B / z_{c}$ is shown in Fig. 3.

$$
\Delta_{B}=\frac{\sqrt{2} z_{c} \delta}{f} \frac{\left(k^{2}+4\right)^{\frac{3}{2}}}{8 k}=\frac{\sqrt{2} z_{c} \delta}{f} e
$$

According to the measurement error curve distribution in Fig. 3, the relation between $e$ and $k=B / z_{c}$ can be described as parabola. Via calculating, when $k=\sqrt{2}, e$ has a min. From the curve, when $k$ vary in $0.7 \sim 2.5$, the error is little, in other occasion, the error is large. So, an ideal system should keep baseline distance and target distance in a same magnitude. But the baseline distance cannot increase unlimitedly, so the structure of the system should change according to actual demand.

\section{Summary}

Based on the above simulation analysis, the structure of the binocular vision system configuration can draw the following conclusions.

(1) When the system is a symmetrical angle structure, it has high measuring accuracy.

(2) When the angle between the optical axis of the baseline value within the range of $30^{\circ} \sim 50^{\circ}$, the measurement error is evenly distributed over the entire field of view. For a close-range target, in order to improve the measurement accuracy, $\alpha$ should be controlled within the range $50^{\circ} \sim 75^{\circ}$. For long-range target, in order to improve measurement accuracy, $\alpha$ should be controlled within the range $15^{\circ} \sim 30^{\circ}$.

(3) In the measuring range, increasing the effective focal length can improve the measurement accuracy, but it will affect the system's observation area. So the relationship between them needs to be balanced in a specific environment.

(4) The ideal system should keep baseline distance and target distance in a same magnitude, but in actual condition, the structure of the system should change to fulfill the accuracy demard.

\section{References}

[1]. Guo Yubo, Yao Yu, Di Xiaoguang. Research on structural parameter optimization of binocular vision measuring system for parallel mechanism. IEEE Translation on Mechatronics and Automation. Vol. 6 (2006) No. 2, p. 1131-1135.

[2]. Meng Huanbiao, Yang Xinqiang, Li Xuemei. Measurement error analysis based on binocular vision system. Fifth National Conference on Geometric Design and Computing. Guangzhou, 2011, p. 141-145.

[3]. Wang Peng, Zhang Qiuzhi, Li Wenqiang, etc. Camera calibration method based on imaging ray tracing space. Journal of Scientific Instrument. Vol. 36 (2015) No. 1, p. 224-229.

[4]. S. J. Maybank. A probabilistic definition of salient regions for image matching. Neurocomputing. Vol. 120 (2013), p. 4-14.

[5]. Wu Zhangliang, Sun Changku, Yang Zhongdong. Analysis and design of structure parameters of vision measuring sensor system. Opto-Electronic Engineering. Vol. 36 (2013) No. 12, p. 56-61, 90.

[6]. An Liansheng. Applied optics. Beijing Institute of Technology Press, 2007, p.45. 